\title{
Detection of Tetracycline in Water Using Glutathione-protected Fluorescent Gold Nanoclusters
}

\author{
Dongri Lu, Xiaoyi Pan, Wei Mu, Chao LI, and Xiaojun Han ${ }^{\dagger}$ \\ State Key Laboratory of Urban Water Resource and Environment, School of Chemistry and Chemical Engineering, \\ Harbin Institute of Technology, 92 West Da-Zhi Street, Harbin 150001, China
}

\begin{abstract}
Tetracycline (Tc), a widely used antibiotic, is one of the major pollutants in water. Herein, glutathione (GSH)-protected $\mathrm{Au}$ nanoclusters (GSH-AuNCs) were prepared to detect Tc. The fluorescence quenching ratio of GSH-AuNCs shows an excellent linear response against tetracycline in the concentration range of $50 \mu \mathrm{g} / \mathrm{L}-50 \mathrm{mg} / \mathrm{L}$ with the detection limit of $5.31 \mu \mathrm{g} / \mathrm{L}$. For the test paper prepared by GSH-AuNCs, $1 \mathrm{mg} / \mathrm{L}$ Tc caused a significant difference that could be recognized by the naked eye. The method exhibited good selectivity and excellent recovery when applied to a tap water sample. The method has the potential for Tc detection in real samples.
\end{abstract}

Keywords Au nanocluster, tetracycline detection, quenching ratio, glutathione, recovery experiment

(Received August 27, 2018; Accepted November 20, 2018; Advance Publication Released Online by J-STAGE November 30, 2018)

\section{Introduction}

Tetracycline (Tc) has been widely used as an antibiotic in the field of veterinary drugs and aquaculture. As a byproduct, it also causes serious environmental problems. It is known that oxytetracycline, a kind of Tcs, does not degrade even after 10 months in marine sediments and soils. ${ }^{1}$ More than half of the applied drugs are not absorbed and remain in the environment, which explains the presence of a high amount of veterinary drugs and their metabolites surrounding aquaculture areas. ${ }^{2}$ Therefore, a rapid method to detect drug residues in water is needed. Although the detection methods for methyl viologen, ${ }^{3}$ organophosphorus ${ }^{4}$ and pesticides ${ }^{5}$ are relatively mature, a rapid detection method for tetracycline is still wanted.

Many analytical methods have been developed to detect Tc, such as high performance liquid chromatography (HPLC), ${ }^{6}$ LC with tandem mass spectrometry (LC-MS), ${ }^{7,8}$ enzyme-linked immunoassay, ${ }^{9,10}$ colorimetric analysis method ${ }^{11,12}$ and electrochemical detection. ${ }^{13,14}$ In general, the methods of LCMS or HPLC have good sensitivity to identify Tc, but these methods involve complicated procedures and the pretreatment of samples takes time. In the field of analysis, attention has increasingly turned to the fluorescence detection method in recent years because of the advantages of ease of operation, low cost, real-time detection, and high sensitivity. ${ }^{15-18}$

Tc, with no fluorescence, was detected by a series of fluorescence sensors in the past few decades with the help of $\mathrm{Eu}^{3+}$-based nanoparticles, ${ }^{19,20}$ metal-organic coordination polymers, ${ }^{21,22}$ CdTe quantum dots, ${ }^{23}$ and carbon quantum dots, ${ }^{24}$ etc. Compared with these fluorescent probes, gold nanoclusters (AuNCs) have low toxicity with an ultra-small size $(\sim 2 \mathrm{~nm})$. Among many protecting agents, glutathione $(\mathrm{GSH})$ is a

† To whom correspondence should be addressed.

E-mail: hanxiaojun@hit.edu.cn (X. H.); muwei@hit.edu.cn (W. M.) tripeptide with good biocompatibility and water-soluble properties. ${ }^{25}$ Therefore, an increasing number of studies have attempted to use glutathione as a protecting agent to synthesize metal nanoclusters. ${ }^{26}$

In this work, gold nanoclusters (GSH-AuNCs), synthesized by the reduction of hydrogen tetrachloroaurate by glutathione, were used as fluorescence probes to detect Tc. Meanwhile AuNCsbased test papers were also developed for detecting Tc by the naked eye.

\section{Experimental}

\section{Materials and apparatus}

Hydrogen tetrachloroaurate $\left(\mathrm{HAuCl}_{4} \cdot 3 \mathrm{H}_{2} \mathrm{O}\right)$ was purchased from Sigma Aldrich (China). Glutathione (GSH) was obtained from Aladdin. Tetracycline was purchased from Macklin (China). Dionized water (DI water) was used throughout the whole experiment. Dopamine was purchased from Sigma (USA), while $\mathrm{Pb}\left(\mathrm{NO}_{3}\right)_{2}, \quad \mathrm{MnCl}_{2} \cdot 4 \mathrm{H}_{2} \mathrm{O}, \quad \mathrm{Hg}\left(\mathrm{NO}_{3}\right)_{2} \cdot 2 \mathrm{H}_{2} \mathrm{O}$, $\mathrm{Ni}\left(\mathrm{NO}_{3}\right)_{2} \cdot 6 \mathrm{H}_{2} \mathrm{O}, \mathrm{CaCl}_{2} \cdot 2 \mathrm{H}_{2} \mathrm{O}, \mathrm{Cd}\left(\mathrm{NO}_{3}\right) \cdot 4 \mathrm{H}_{2} \mathrm{O}, \mathrm{Mg}\left(\mathrm{NO}_{3}\right)_{2} \cdot 6 \mathrm{H}_{2} \mathrm{O}$, $\mathrm{KCl}, \mathrm{Ni}\left(\mathrm{NO}_{3}\right)_{2} \cdot 6 \mathrm{H}_{2} \mathrm{O}, \mathrm{Na}_{2} \mathrm{SO}_{4}, \mathrm{ZnSO}_{4}$, citric acid, $\mathrm{NH}_{4} \mathrm{Cl}$, glucose and EDTA-2Na were all provided by Tianjin Fengchuan Chemical Reagent Co. (Tianjin, China).

\section{Measurements}

The high-resolution transmission electron microscope (HRTEM) characterization was measured by a JEM-2100 transmission electron microscope under $200 \mathrm{kV}$ accelerating voltage. Fluorescence spectra were obtained on an LS55 luminescence spectrometer (PerkinElmer, UK). Ultravioletvisible (UV-vis) absorption spectroscopy was measured with a Cary $60 \mathrm{UV} /$ Vis spectrometer (Agilent Technologies, USA). Zeta potential was recorded on a Zetasizer Nano (Malvern Instruments Ltd., UK). 

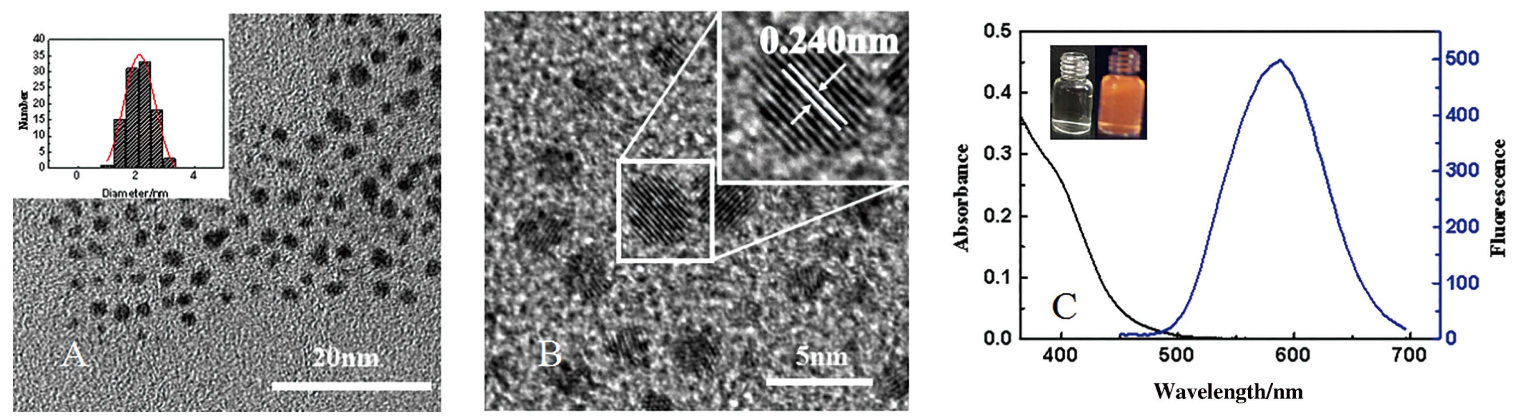

Fig. 1 HR-TEM images (A, B) of GSH-AuNCs and single AuNC. The inset of A shows the size distributions of GSH-AuNCs. (C) (a) UV-vis absorption spectrum and (b) photoluminescent spectrum (ex $=365 \mathrm{~nm}$ ) of prepared AuNCs. The inset shows the photographs of AuNCs solution under room light (left) and UV-lamp (right), respectively.

\section{Synthesis of GSH-AuNCs}

GSH functionalized AuNCs were synthesized by following the procedure described in a previous paper. ${ }^{27}$ The steps were: 1) $2.0 \mathrm{~mL} \mathrm{HAuCl}_{4}$ with the concentration of $20 \mathrm{mM}$ was added to $17.4 \mathrm{~mL}$ DI water, 2) $0.0184 \mathrm{~g}$ GSH was added to $0.60 \mathrm{~mL}$ DI water, 3) the abovementioned solutions were mixed together at $25^{\circ} \mathrm{C}$ for $5 \mathrm{~min}, 4$ ) after gentle stirring for $24 \mathrm{~h}$ under $70^{\circ} \mathrm{C}$, a solution of orange-emitting GSH-AuNCs was obtained. The prepared GSH-AuNCs were kept at around $4^{\circ} \mathrm{C}$.

Determination of Tc in aqueous solution by AuNCs

In order to measure the concentration of $\mathrm{Tc}$ in aqueous solution, the aliquots of $1 \mathrm{~mL}$ of GSH-AuNCs $(0.5 \mathrm{mM})$ were added into several tubes. Next, $1 \mathrm{~mL}$ of Tc solution with different contentrations in the range of $50 \mu \mathrm{g} / \mathrm{L}-50 \mathrm{mg} / \mathrm{L}$ were added to each tube. All the solutions were diluted with buffer solution, meanwhile, to avoid the effect of $\mathrm{pH}$ change. The control experiments were carried out by adding a certain amount of dopamine, $\mathrm{Ni}^{2+}, \mathrm{Zn}^{2+}, \mathrm{Mn}^{2+}, \mathrm{Ca}^{2+}, \mathrm{Mg}^{2+}$, citric acid, glucose, $\mathrm{NH}_{4}^{+}, \mathrm{K}^{+}, \mathrm{Na}^{+}$, and EDTA-2Na.

\section{Detection of Tc based on AuNCs-modified test papers}

The filter paper was cut into circles with a diameter of $1 \mathrm{~cm}$, and immersed into the previously prepared GSH-AuNCs solution for $2 \mathrm{~h}$, then dried in a closed environment. A certain amount of Tc sample $(20 \mu \mathrm{L})$ was dropped onto the filter paper modified with AuNCs. From the change of the fluorescence intensity of the test paper, the concentration of tetracycline could be roughly determined.

\section{Results and Discussion}

\section{Characterization of GSH-AuNCs}

The black dots in Fig. 1A are gold nanoclusters. From high resolution images in Fig. 1B, the lattice spacing can be easily discerned. A single GSH-AuNC is shown in the inset of Fig. 1B, in which the lattice spacing of GSH-AuNC is $0.240 \mathrm{~nm}$. The data is consistent with the primary reflection of the (111) lattice of $\mathrm{Au} \mathrm{NCs}$ and confirms the crystalline nature of $\mathrm{Au} .^{28}$ The inset of Fig. 1A shows the size distributions of GSHAuNCs; the majority of AuNCs are in the range of 1 to $3 \mathrm{~nm}$ with an average diameter of $2.3 \mathrm{~nm}$.

Figure 1C shows the photoluminescent (PL) spectrum and UV-vis absorption spectrum of the GSH-AuNCs. In the absorption spectrum, there is a shoulder peak at $\sim 400 \mathrm{~nm}$, which

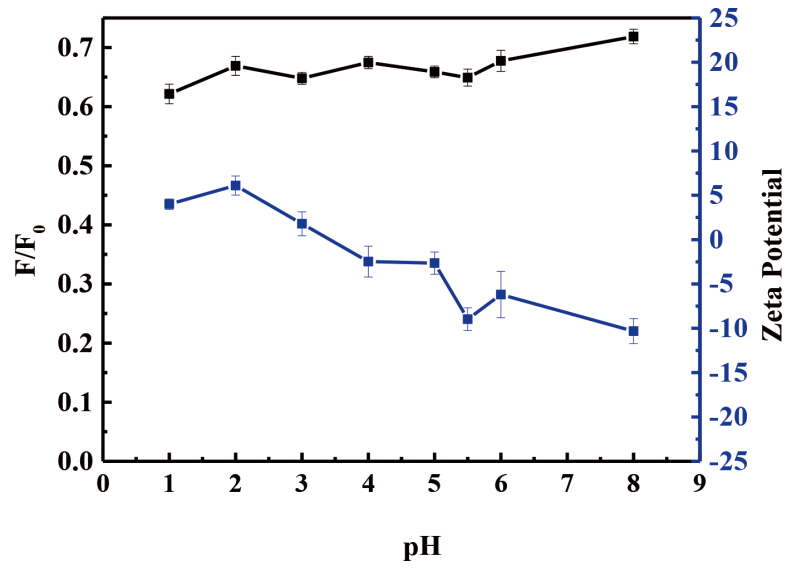

Fig. 2 Effect of $\mathrm{pH}$ on fluorescence intensity of GSH-AuNCs.

means the prepared AuNCs have the size of $\sim 2.3 \mathrm{~nm}$ since neither surface plasmon resonance (SPR) nor molecular absorption was detected. ${ }^{29}$ The main emission peak of the GSHAuNCs is about 590 with $365 \mathrm{~nm}$ excitation wavelength (blue curve in Fig. 1C). The synthesized GSH-AuNCs solution is light yellow under natural light and orange under UV light (the inset in Fig. 1C). The above data confirm that the GSH-AuNCs have been successfully synthesized.

\section{Tc detection}

The prepared AuNCs were then used for Tc detection. To investigate the effect of $\mathrm{pH}$ on $\mathrm{Tc}$ detection, $\mathrm{pH}$ titration of GSH-AuNCs was performed. The value of $F / F_{0}$ is used to indicate the fluorescence quenching degree of GSH-AuNCs, where $F_{0}$ is the initial fluorescence intensity of GSH-AuNCs, and $F$ means the fluorescence intensity of GSH-AuNCs after adding and reacting with a certain concentration of Tc. The value of $F / F_{0}$ showed no significant change in the $\mathrm{pH}$ range of 1 to 8 (black curve in Fig. 2), which implies pH has no obvious effect on the fluorescence intensity of GSH-AuNCs. The zeta potential of GSH-AuNCs was measured to determine the isoelectric point of GSH-AuNCs. From the blue curve in Fig. 2, it is noted that the isoelectric point of GSH-AuNCs is between $\mathrm{pH} 3$ and 4. Based on the isoelectric point of Tc of $\mathrm{pH} 5.5,{ }^{30}$ we decided to use $\mathrm{pH}$ of 5 in order to enable the electrostatic interaction between AuNCs and Tc molecules for Tc detection. 

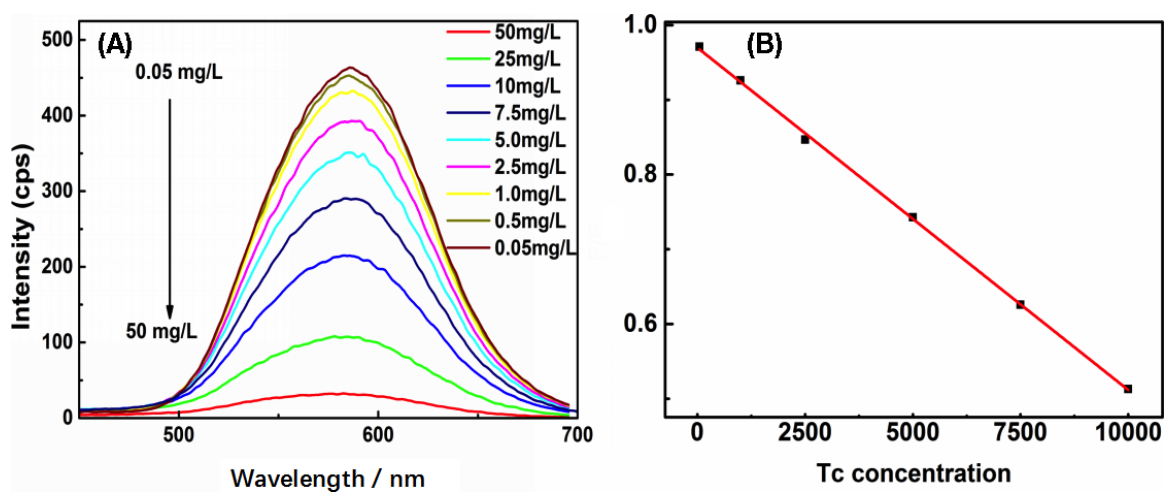

Fig. 3 (A) Fluorescence emission spectra of the GSH-AuNCs in the presence of increasing Tc concentrations $(0.05-50 \mathrm{mg} / \mathrm{L})$. (B) The linear plot of $F / F_{0}$ at $590 \mathrm{~nm}$ as a function of the Tc concentration $(0.05-10 \mathrm{mg} / \mathrm{L})$.

Table 1 Comparison of the proposed method with other reported methods of Tc fluorescence detection in recent years

\begin{tabular}{|c|c|c|c|c|}
\hline $\begin{array}{l}\text { Analytical } \\
\text { method }\end{array}$ & $\begin{array}{c}\text { Reaction } \\
\text { medium }\end{array}$ & $\begin{array}{l}\text { Detection } \\
\text { limit/ } / \mu \mathrm{g} / \mathrm{L}\end{array}$ & $\begin{array}{c}\text { Enhancement } \\
\text { reagent }\end{array}$ & Ref. \\
\hline $\begin{array}{l}\text { Flow injection } \\
\text { analysis }\end{array}$ & $\begin{array}{c}\text { Aqueous } \\
\text { solution }\end{array}$ & 0.03 & No & 31 \\
\hline Chemiluminescence & $\begin{array}{l}\text { Aqueous } \\
\text { solution }\end{array}$ & 22.2 & No & 32 \\
\hline Fluorescence & $\begin{array}{r}\text { Aqueous } \\
\text { solution }\end{array}$ & 8.88 & $\mathrm{AgNPs}+\mathrm{Eu}^{3+}$ & 18 \\
\hline Fluorescence & $\begin{array}{l}\text { Aqueous } \\
\text { solution }\end{array}$ & 20 & $\mathrm{CdTe}$ & 33 \\
\hline Fluorescence & $\begin{array}{l}\text { Aqueous } \\
\text { solution }\end{array}$ & 5.31 & AuNCs & This work \\
\hline
\end{tabular}

Different concentrations of tetracycline were added to gold nanocluster solutions of equal volume to observe the fluorescence quenching effect, as shown in Fig. 3. The fluorescent spectra of the samples (Fig. 3A) show that the fluorescence intensity at $590 \mathrm{~nm}$ decreased with the Tc concentration increasing from 0.05 to $50 \mathrm{mg} / \mathrm{L}$, which means that the fluorescence intensity of GSH-AuNCs is highly correlated with the concentration of Tc. A good linear relationship was fitted between the $F / F_{0}$ and Tc concentration $\left(R^{2}=0.99\right)$ in the range of $50 \mu \mathrm{g} / \mathrm{L}$ to $10 \mathrm{mg} / \mathrm{L}$ (as shown in Fig. 3B) with the detection limit of $5.31 \mu \mathrm{g} / \mathrm{L}$ at a signal-to-noise ratio of 3 . Table 1 shows a comparison of the fluorescence detection of tetracycline reported in papers published in recent years, and indicates our method has good sensitivity for Tc detection.

The mechanism of how GSH-AuNCs respond to Tc has been investigated. The zeta potential of GSH-AuNCs before and after addition of Tc was compared. An obvious change (from -6.27 to $-5.40 \mathrm{mV}$ ) of the zeta potential occured when $\mathrm{pH} 5$, which indicated the strong electrostatic interaction between AuNCs and Tc. Therefore, we make a hypothesis about the mechanism for the fluorescence quenching phenomenon. It is more likely that the electron transfer occurs between the tetracycline and gold nanoclusters due to electrostatic interactions.

AuNCs probes were also studied for the selectivity of Tc detection. Different kinds of organics and metal ions were added into the GSH-AuNCs, such as dopamine, $\mathrm{Ni}^{2+}, \mathrm{Zn}^{2+}$, $\mathrm{Mn}^{2+}$, EDTA-2Na etc., to evaluate the selectivity. Here, $50 \mathrm{mg} / \mathrm{L}$

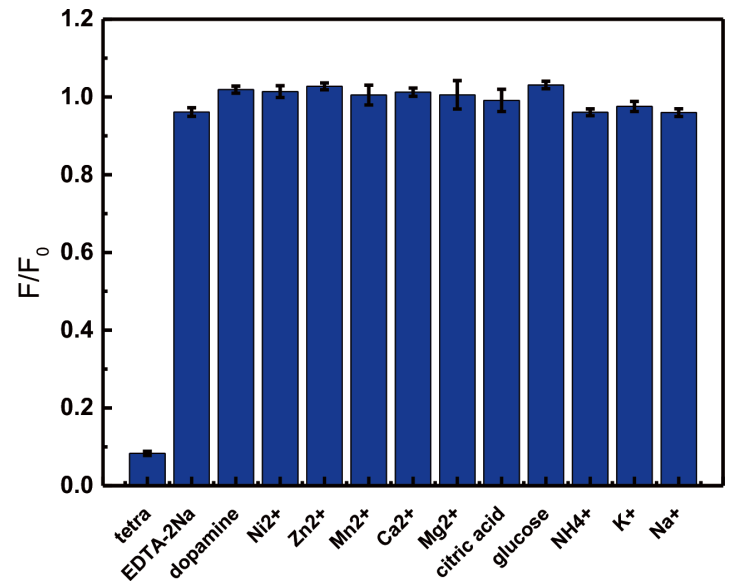

Fig. 4 Selectivity of the GSH-AuNCs in the presence of $50 \mathrm{mg} / \mathrm{L} \mathrm{Tc}$ or $5 \mathrm{~g} / \mathrm{L}$ of another analyte. $F_{0}$ and $F$ are the fluorescence intensity of GSH-AuNCs in the absence and presence of Tc and the other analytes, respectively.

Tc solution was added into the solution of GSH-AuNCs, while the concentration of interfering substances was 100 times that of Tc. As shown in Fig. 4, the corresponding metal ions and organics $F / F_{0}$ value are all about equal to 1 , which means these substances do not interfere with the detection or affect the fluorescence intensity of GSH-AuNCs. GSH-AuNCs have excellent selectivity for tetracycline among those interfering substances. These results clearly show that AuNCs have the ability to specifically identify Tc.

\section{Detection of Tc in real samples}

In order to evaluate real application ability of this method, it was used for the analysis of Tc in pure water and tap water samples. In short, different amounts of Tc were added to equal volume samples of pure water and tap water, respectively. The Tc concentrations detected in the tap water samples were calculated using standard curves and regression equations to obtain the corresponding recoveries and RSD. Importantly, as listed in Table S1 (Supporting Information), the average recovery in pure water and tap water samples performed by the standard addition method and the RSD were generally satisfactory.

Also, GSH-AuNCs-based test papers were prepared to detect Tc. 


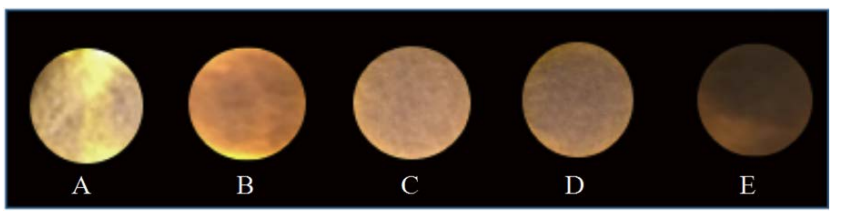

Fig. 5 The GSH-AuNCs based test papers dropped with different concentrations of Tc. Dropped with water (A), $1 \mathrm{mg} / \mathrm{L} \mathrm{Tc}$ (B), $2.5 \mathrm{mg} / \mathrm{L} \mathrm{Tc}(\mathrm{C}), 5 \mathrm{mg} / \mathrm{L} \mathrm{Tc}(\mathrm{D})$, and $50 \mathrm{mg} / \mathrm{L} \mathrm{Tc}(\mathrm{E})$.

The samples containing different concentrations of Tc were dropped on the test paper and the images were observed under the UV lamp (Fig. 5). As seen in Fig. 5, different concentrations of Tc from 1 to $50 \mathrm{mg} / \mathrm{L}$ (Figs. 5B - 5E) result in a clear visible fluorescent quenching effect, which can be observed by the naked eye. Meanwhile, the same amount of DI water was dropped on test paper as a control. The test paper can detect as low as $1 \mathrm{mg} / \mathrm{L}$ Tc by the naked eye. Therefore, the present method has the potential of practical application for detecting Tc in real water samples.

\section{Conclusions}

In this study, GSH-AuNCs were synthesized as fluorescent probes to detect tetracycline by the quenching effect of tetracycline to the GSH-AuNCs. The detection limit was determined to be $5.31 \mu \mathrm{g} / \mathrm{L}$. A portable AuNCs-based filter paper was also prepared by soaking filter paper in AuNCs solution. The difference can be easily distinguished by the naked eye when $1 \mathrm{mg} / \mathrm{L}$ tetracycline was dropped on the test paper. With good sensitivity and selectivity, our method represents a promising approach to detect Tc in real samples.

\section{Acknowledgements}

This work was supported by the National Key R\&D Program of China (2016YFC0401104). HIT Environment and Ecology Innovation Special Funds (HSCJ201617), and the National Natural Science Foundation of China (Grant Nos. 21773050 and 21528501).

\section{Supporting Information}

Supporting information includes a table exhibiting the recovery of Tc in pure water and real water samples detected by the current method. This material is available free of charge on the Web at http://www.jsac.or.jp/analsci/.

\section{References}

1. M. Rabølle and N. H. Spliid, Chemosphere, 2000, 40, 715.

2. S. C. Kim and K. Carlson, Water Res., 2006, 40, 2549.

3. Y. Su, S. Ma, K Jiang, and X. Han, Chin. J. Chem., 2015, $33,446$.

4. H. Wang, Y. Su, H. Kim, D. Yong, L. Wang, and X. Han,
Chin. J. Chem., 2015, 33, 1135.

5. H. Ma, D. Yong, H. Kim, Z. Zhang, S. Ma, and X. Han, Electroanalysis, 2016, 28, 580.

6. J. Song, Z. H. Zhang, Y. Q. Zhang, C. Feng, G. N. Wang, and J. P. Wang, Anal. Methods, 2014, 6, 6459.

7. C. Nebot, M. Guarddon, F. Seco, A. Iglesias, J. M. Miranda, C. M. Franco, and A. Cepeda, Food Control, 2014, 46, 495.

8. Q. Zhou, Y. Zhang, N. Wang, L. Zhu, and H. Tang, Food Control, 2014, 46, 324.

9. S. Wang, W. Yong, J. Liu, L. Zhang, Q. Chen, and Y. Dong, Biosens. Bioelectron., 2014, 57, 192.

10. S. Wang, J. Liu, W. Yong, Q. Chen, L. Zhang, Y. Dong, H. Su, and T. Tan, Talanta, 2015, 131, 562.

11. Y. S. Kwon, N. H. Ahmad Raston, and M. B. Gu, Chem. Commun., 2014, 50, 40.

12. L. Shen, J. Chen, N. Li, P. He, and Z. Li, Anal. Chim. Acta, 2014, 839, 83.

13. D. Vega, L. Agüí, A. González-Cortés, P. Yáñez-Sedeño, and J. M. Pingarrón, Anal. Bioanal. Chem., 2007, 389, 951.

14. C. M. F. Calixto and É. T. G. Cavalheiro, Anal. Lett., 2015, 48, 1454.

15. Y. Cheng, Y. Zhang, R. Pei, Y. Xie, W. Yao, Y. Guo, and H. Qian, Anal. Sci., 2018, 34, 415.

16. B. G. Abraham, V. Santala, N. V. Tkachenko, and M. Karp, Anal. Bioanal. Chem., 2014, 406, 7195.

17. H. S. Jung, P. S. Kwon, J. W. Lee, J. I. Kim, S. H. Chang, J. W. Kim, S. Yan, Y. L. Jin, J. H. Lee, and T. Joo, J. Am. Chem. Soc., 2009, 131, 2008.

18. N. Abe and N. Iki, Anal. Sci., 2017, 33, 1141.

19. H. Tan, Y. Chen, and Y. Chen, Sens. Actuators, B, 2012, 173, 262.

20. X. Yang, S. Zhu, D. Yao, Z. Yan, Y Luo, and Y. Feng, Talanta, 2014, 122, 36.

21. F. Leng, X. J. Zhao, J. Wang, and Y. F. Li, Talanta, 2013, 107, 396.

22. H. Tan, C. Ma, Y. Song, F. Xu, S. Chen, and W. Li, Biosens. Bioelectron., 2013, 50, 447.

23. C. Gao, Z. Liu, J. Chen, and Z. Yan, Luminescence, 2013, 28,378

24. J. Hou, H. Li, L. Wang, P. Zhang, T. Zhou, H. Ding, and L. Ding, Talanta, 2015, 146, 34.

25. J. Yang, N. Xia, X. Wang, X. Liu, A. Xu, Z. Wu, and Z. Luo, Nanoscale, 2015, 7, 18464.

26. C. T. Chen, W. J. Chen, C. Z. Liu, L. Y Chang, and Y. C. Chen, Chem. Commun., 2009, 48, 7515.

27. R. X. Bian, X. T. Wu, F. Chai, L. Li, L. Y. Zhang, T. T. Wang, C. G. Wang, and Z. M. Su, Sens. Actuators, B, 2017, 241, 592.

28. X. Yang, S. Zhu, Y. Dou, Y. Zhuo, Y. Luo, and Y. Feng, Talanta, 2014, 122, 36.

29. S. Link and M. S. El-Sayed, Annu. Rev. Phys. Chem., 2003, 54,331

30. C. R. Anderson, H. S. Rupp, and W. H. Wu, J. Chromatogr. A, 2005, 1075, 23.

31. J. M. Traviesa-Alvarez, J. M. Costa-Fernández, R. Pereiro, and A. Sanz-Medel, Anal. Chim. Acta, 2007, 589, 51.

32. M. Kaczmarek, A. Idzikowska, and S. Lis, J. Fluoresc., 2008, 18, 1193.

33. J. Hou, J. Yan, Q. Zhao, Y. Li, H. Ding, and L. Ding, Nanoscale, 2013, 5, 9558. 